RECOMENDACIONES

\title{
RECOMENDACIONES PARA EL MANEJO DE LAS PACIENTES CON DIABETES PREGESTACIONAL
}

\author{
RECOMMENDATIONS FOR THE MANAGEMENT \\ OF PATIENTS WITH PREGESTATIONAL DIABETES
}

\author{
María Gabriela Rovira ${ }^{1}$, Alicia Jawerbaum², Liliana Glatstein ${ }^{3}$, Stella Sucani ${ }^{4}$, Celina Bertona ${ }^{5}$, \\ Inés Argerich ${ }^{6}$, Carolina Gómez Martí , María Vivona ${ }^{8}$, Verónica Kojdamanian ${ }^{9}$, Silvina Guaitas ${ }^{10}$, \\ Eugenia Capobianco ${ }^{2}$, Beatriz Villarroel Parra ${ }^{11}$, Eugenia Rivas ${ }^{12}$, María Elena Rodríguez ${ }^{13}$ \\ Asesores: Jorge Alvariñas ${ }^{14}$, Susana Salzberg ${ }^{15}$, María Cristina Faingold ${ }^{16}$, Silvia Lapertosa ${ }^{17}$, Natalia Basualdo ${ }^{18}$
}

\section{RESUMEN}

La diabetes mellitus pregestacional afecta el desarrollo de la gestación y se asocia a complicaciones maternas y feto-neonatales. Si bien hace algunos años la mayoría de las pacientes con diabetes pregestacional era diabética tipo 1, la prevalencia de mujeres con diabetes tipo 2 en edad reproductiva aumentó asociada al incremento de la obesidad. La paciente con diabetes pregestacional con mal control presenta mayor riesgo de complicaciones desde el inicio y hasta el final de embarazo.

El control metabólico adecuado, el correcto estado nutricional y el tratamiento de las complicaciones maternas previenen las complicaciones o reducen su severidad. Por ello es fundamental la programación del embarazo para prevenir el daño en las primeras semanas de gestación. Además es necesario el riguroso seguimiento clínico durante todo el embarazo.

En este contexto, en el marco del Comité de Diabetes y Embarazo, y a través del estudio de evidencias científicas y estudios vinculados, surgen estas recomendaciones para mujeres con diabetes pregestacional.

Palabras clave: diabetes, embarazo, diabetes pregestacional.

\section{ABSTRACT}

Pregestational diabetes mellitus impairs development during gestation and is associated with maternal and fetal-neonatal complications. Although a few years ago most of the patients with pregestational diabetes were type 1 diabetics, the prevalence of women with type 2 diabetes in reproductive age has increased, associated with an increase in obesity. Patients with poorly controlled pregestational diabetes have a higher risk of complications from the onset to the end of pregnancy. Adequate metabolic control, proper nutritional status, and treatment of maternal complications prevent complications or reduce their severity. For this reason, pregnancy programming is essential to prevent damage in the first weeks of gestation. In addition, rigorous clinical follow-up is required throughout pregnancy.

In this context and through the study of scientific evidences and related studies, the Diabetes and Pregnancy Committee propose these recommendations for women with pregestational diabetes.

Key words: diabetes, pregnancy, pregestational diabetes.

Revista de la Sociedad Argentina de Diabetes 2017; Vol. 51 (153-174)
1 Coordinadora del Comité de Diabetes y Embarazo de la Sociedad Argentina de Diabetes (SAD), Médica Endocrinóloga del Hospital Británico de Buenos Aires, CABA, Argentina

2 Universidad de Buenos Aires (UBA), Facultad de Medicina, CONICET-UBA, Laboratorio de Reproducción y Metabolismo, CEFYBO, CABA, Argentina

3 Ex Jefa del Servicio de Clínica Médica del Hospital Materno Provincial de Córdoba, Facultad de Medicina Universidad Nacional del Nordeste (UNNE), Entre Ríos, Argentina

4 Jefa del Servicio de Clínica Médica del Hospital Materno Provincial Dr. Raúl Felipe Lucini, Córdoba, Argentina

5 Hospital Universitario UNCuyo, Mendoza, Argentina

6 Encargada de la Unidad de Diabetes y Embarazo del Hospital Perrupato, San Martín, Mendoza, Argentina

7 Co-directora del Centro Integral de Endocrinología y Diabetes (Cendia) Concordia, Entre Ríos, Argentina

8 Jefa de la Unidad del Servicio de Clínica Médica del Hospital Santojanni, a cargo del consultorio de Diabetes y Embarazo, CABA, Argentina

9 Centro Médico Pilar, Prov. de Bs. As., Argentina

10 Servicio de Nutrición y Diabetes del Hospital Universitario Austral, Prov. de Bs. As., Argentina
11 Médica especialista en Nutrición Clínica (UBA), Hospital Vélez Sarsfield, Sanatorio Santa Isabel, CABA, Argentina

12 Especialista en Obstetricia y Ginecología, Médica de Planta del Servicio de Obstetricia, Hospital Materno Infantil Ramón Sardá, CABA, Argentina

13 Encargada de Clínica y Diabetes del sector de alto riesgo del Hospital Materno Infantil de San Isidro, Hospital Central de San Isidro, Prov. de Bs. As., Argentina

14 Médico Consultor en Nutrición Hospital Enrique Tornú, ex Presidente de la Sociedad Argentina de Nutrición, ex presidente de la SAD, Miembro Honorario de la Asociación Latinoamericana de Diabetes (ALAD), CABA, Argentina

15 Directora de la Escuela de Graduados (1999-2000), Coordinadora del Grupo de Trabajo ALAD (2013-2016), CABA, Argentina

16 Jefa del Servicio de Endocrinología de la Unidad Asistencial Dr. César Milstein, Directora de la carrera de Médico Especialista en Endocrinología (UBA), CABA, Argentina

17 Facultad de Medicina UNNE, Subdelegada Argentina de ALAD, Entre Ríos, Argentina

18 Jefa del Servicio de Tocoginecología del Sanatorio Anchorena, CABA, Argentina 
Contacto del autor: María Gabriela Rovira

E-mail: mgrovira1@gmail.com

Correspondencia: Libertador 4992 PB $1^{\circ}$ (C1426BWX),

CABA, Argentina

Tel.: (011) 4772-4391
Fecha de trabajo recibido: 30/07/17

Fecha de trabajo aceptado: 29/09/17

Conflictos de interés: los autores declaran que no existe conflicto de interés

\section{INTRODUCCIÓN}

La diabetes mellitus pregestacional afecta el desarrollo de la gestación y se asocia a complicaciones maternas y feto-neonatales. Si bien hace algunos años la mayoría de las pacientes con diabetes pregestacional era diabética tipo 1 (DM1), la prevalencia de mujeres con diabetes tipo 2 (DM2) en edad reproductiva aumentó asociada al incremento de la obesidad. Debemos considerar que el embarazo de una mujer con diabetes es un embarazo de riesgo. La paciente con diabetes pregestacional con mal control presenta mayor riesgo de complicaciones desde el inicio y hasta el final del embarazo. Los mecanismos de inducción que subyacen a estas alteraciones incluyen procesos metabólicos aberrantes, generación de un entorno prooxidante y proinflamatorio, cambios patológicos en las vías de señalización y cambios epigenéticos que se vincularán a procesos patológicos que podrán ser evidentes a lo largo de la vida.

El control metabólico adecuado, el correcto estado nutricional y el tratamiento de las complicaciones maternas previenen las complicaciones o reducen su severidad. Por ello, es fundamental la programación del embarazo para prevenir el daño en las primeras semanas de gestación.

Además es necesario el riguroso seguimiento clínico durante todo el embarazo. El tratamiento requerirá modificaciones para acompañar los importantes cambios metabólicos propios de la fisiología del embarazo y su adecuada respuesta será crucial en el cuidado de la salud materna, el desarrollo feto-placentario, el período neonatal y la prevención de patologías futuras en el hijo y la madre.

En este contexto, en el marco del Comité de Diabetes y Embarazo, y a través del estudio de evidencias científicas y estudios vinculados, surgen estas recomendaciones para mujeres con diabetes pregestacional.

La fuerza de las recomendaciones y la calidad de la evidencia se basan en el sistema Grading of Recomendations Assessment, Development and Evaluation (GRADE), en el cual se informa la calidad de la evidencia: "alta", "moderada", "baja” o "muy baja", en vinculación a la evidencia (ensayos clínicos, estudios observacionales u otras evidencias), y se suma la fuerza de la recomendación: "fuerte" o "débil", y "a favor" (cuando se recomienda su práctica en los pacientes) o "en contra" (cuando se recomienda no realizar dicha práctica y por lo tanto no debiera usarse en los pacientes) 1.

\section{Objetivos}

El objetivo de estas recomendaciones es actualizar las ya publicadas e incorporar novedades y modificaciones de utilidad para los profesionales de la salud que atienden pacientes diabéticas embarazadas.

\section{Diabetes pregestacional (DPG)}

Definimos como DPG:

- A una mujer con diabetes tipo 1 ó 2 que se embaraza.

- Embarazada que cumple con los criterios de diagnóstico de diabetes de la Organización Mundial de la Salud (OMS) durante el primer trimestre del embarazo:

- Síntomas clásicos de diabetes (polidipsia, poliuria, polifagia y bajo peso) y una glucemia en cualquier momento del día mayor o igual a $200 \mathrm{mg} / \mathrm{dl}$, sin relación con el tiempo transcurrido desde la última comida.

- Glucemia en ayunas en plasma venoso mayor o igual a $126 \mathrm{mg} / \mathrm{dl}$ confirmada con un segundo test realizado en el laboratorio en el curso de la semana.

- Glucemia mayor o igual a 200 mg/dl dos horas después de una carga de $75 \mathrm{~g}$ de glucosa durante una prueba de tolerancia a la glucosa oral (PTGO).

\section{A) CUIDADOS PRECONCEPCIONALES}

\section{Consulta preconcepcional (ver resumen en Anexo I)}

\section{Programación del embarazo}

Se define como las intervenciones realizadas antes del embarazo, cuyo objetivo es evaluar y tratar los factores de riesgo que puedan afectar la salud fetal o materna.

Si bien con el avance de los recursos terapéuticos el control metabólico mejoró y determinó una 
mejoría en el control metabólico y consecuentemente disminuyó el riesgo de complicaciones en mujeres con diabetes, este riesgo no ha igualado a la población general. En este contexto la programación del embarazo reduce significativamente las complicaciones a corto y a largo plazo.

\section{Recomendaciones}

- Explicar a la paciente la importancia de planificar el embarazo para disminuir los riesgos de complicaciones maternas y fetales.

- Consensuar con la pareja un método anticonceptivo hasta lograr el objetivo propuesto (Anexo II).

- Informar a las mujeres que planifican un embarazo y tienen diabetes sobre la posible repercusión en la evolución de su enfermedad y en los resultados perinatales.

- Realizar una detallada anamnesis para identificar los posibles riesgos y patologías asociadas.

- Trabajar con un equipo multidisciplinario para el manejo de las posibles complicaciones asociadas, siempre de manera individualizada para cada paciente.

Fuerza de las recomendaciones: alta. Calidad de la evidencia: fuerte a favor.

\section{Objetivos a alcanzar}

- Correcto control del metabolismo hidrocarbonado y lipídico.

- Normalizar el estado nutricional.

- Evaluar:

- Patología cardiovascular.

- Microvascular: retinopatía y nefropatía.

- Evaluación tiroidea.

- Hábitos: alimentarios, tabaquismo, drogadicción y automedicación.

- Medicación de riesgo para el embarazo.

\section{Objetivos del control metabólico}

Estudios de observación demuestran un aumento del riesgo de embriopatía diabética y abortos espontáneos, asociados a elevaciones de la $\mathrm{HbA} 1 \mathrm{c}$ durante las primeras ocho semanas de embarazo y apoyan la recomendación de optimizar el control glucémico previo a la concepción.

\section{Recomendaciones}

- Aconsejar a las mujeres con diabetes en edad fértil acerca de la importancia del buen control metabólico antes de la concepción.

- El objetivo de la HbA1c pregestacional es menor a $7 \%(53 \mathrm{mmol} / \mathrm{mol})$ con mínima cantidad de hipoglucemias.

Fuerza de las recomendaciones: alta. Calidad de la evidencia: fuerte a favor.

\section{Laboratorio preconcepcional}

En las visitas de asesoramiento antes de la concepción se recomienda determinar:

- Serología para rubéola, enfermedad de Chagas, VDRL (por su siglas en inglés, Venereal Disease Research Laboratory), toxoplasmosis, hepatitis B y VIH.

- Glucemia, perfil lipídico, tipificación sanguínea (grupo y factor Rh) y hemograma.

- HbA1c.

- Pruebas de función renal: creatinina, urea, relación de albúmina/creatinina en la orina (RAC), clearance de creatinina y urocultivo.

- Considerar el rastreo de enfermedad celíaca y de patología tiroidea.

\section{Evaluación de complicaciones crónicas}

- Considerar en pacientes de riesgo una evaluación cardiovascular.

- Analizar la función renal para identificar o descartar la presencia de nefropatía diabética.

- Evaluar la presencia de retinopatía diabética, diagnóstico, tratamiento y confirmación de estabilidad de la lesión previo a la concepción.

\section{Evaluación nutricional}

Se recomienda:

- Acordar el plan alimentario según hábitos, actividad física y posibilidad económica.

- Dirigir el plan alimentario para mantener un peso saludable (IMC 20 a 25) o para descender de peso en caso que la paciente presente sobrepeso u obesidad. En aquellas que no lo logren, explicar el incremento del riesgo de malformaciones congénitas, aborto espontáneo, macrosomía, cesárea, hipertensión, preeclampsia, prematurez y mortalidad perinatal.

- Considerar el fraccionamiento de los hidratos de carbono complejos y optar por un régimen de 
insulinoterapia intensificado según el plan de alimentación prescripto

- Suplemento de ácido fólico de 5 mg/día.

\section{Recomendación}

Iniciar el embarazo con un peso adecuado dado que se ha demostrado que a mayor índice de masa corporal (IMC) preconcepcional es mayor el riesgo de malformaciones congénitas, preeclampsia e hipertensión gestacional.

Fuerza de las recomendaciones: alta. Calidad de la evidencia: fuerte a favor.

\section{Evaluación de la medicación preconcepcional}

- Se recomienda suspender el uso de drogas desaconsejadas durante el embarazo en el período preconcepcional:

- Inhibidores de la enzima convertidora de la angiontensina (IECA).

- Antagonistas de receptores de la angiotensina II (ARA II).

- Estatinas.

- Fibratos.

- Indicar la abstención de tabaco, alcohol, drogas ilícitas y abuso de fármacos en general.

- Insulinas. Se sugiere que desde esta etapa la paciente utilice los tipos de insulina que mantendrá durante el embarazo.

- Las insulinas NPH y regular o cristalina y los análogos detemir y aspart están autorizados por la Administración Nacional de Medicamentos, Alimentos y Tecnología Médica (ANMAT) para su utilización en el embarazo.

- Los análogos glulisina, lispro, glargina y degludec no se recomiendan porque no están autorizados por las entidades regulatorias para su utilización en el embarazo.

- Se recomienda iniciar tratamiento con insulina en mujeres tratadas con hipoglucemiantes orales ya que los mismos no están aprobados por las entidades regulatorias locales para su uso en la gestación.

- En pacientes con síndrome de ovario poliquístico se ha demostrado que la utilización de metformina podría ser una alternativa de tratamiento válida ya que disminuye la tasa de abortos espontáneos.

\section{Evaluación ginecológica y de antecedentes obstétricos y reproductivos}

Se recomienda:

- Realizar una evaluación ginecológica completa previa a la concepción (citología cervical -PAP-, examen mamario y anamnesis).

- Evaluar los siguientes antecedentes:

- Muerte perinatal en embarazo anterior. Antecedente de recién nacido con malformaciones del tubo neural.

- Amenaza de parto prematuro en embarazos anteriores.

- Recién nacido con peso al nacer de $<2.500 \mathrm{~g}$ ó $\geq a$ $4.000 \mathrm{~g}$.

- Aborto habitual o provocado.

- Cirugía uterina anterior.

- Hipertensión.

- Diabetes gestacional en embarazos anteriores.

- Nuliparidad o multiparidad.

- Intervalo intergenésico <de 2 años.

\section{Evaluación odontológica}

Se recomienda la evaluación odontológica preconcepcional para la detección de patología bucal (periodontitis, caries) que pueda vincularse a un estado proinflamatorio y a patologías gestacionales.

\section{Actividad física}

Durante la programación del embarazo se recomienda la práctica habitual de ejercicio personalizado dado que favorece el mejor control metabólico.

\section{Vacunación}

Se recomienda verificar el esquema de vacunación en la etapa preconcepcional y completarlo de ser necesario (recordar que si se administra la vacuna para rubéola debe mantenerse el método anticonceptivo por tres meses).

\section{Se recomienda desaconsejar el embarazo} si la paciente presenta:

- Obesidad grado IV.

- Enfermedad coronaria no revascularizada.

- Insuficiencia renal: clearance de creatinina $<50 \mathrm{ml} / \mathrm{min}$ o creatinina sérica $>2 \mathrm{mg} / \mathrm{dl}$.

- Hipertensión arterial que no mejore con la terapéutica medicamentosa permitida en el embarazo.

- Retinopatía proliferativa activa que no responde a la panfotocoagulación.

- Gastroenteropatía diabética severa. 


\section{B) CUIDADOS EN EL EMBARAZO}

\section{1) Seguimiento clínico (ver resumen en Anexo III)}

El control y seguimiento clínico durante el embarazo no difieren en los distintos tipos de pacientes con diabetes. Para reducir el riesgo de complicaciones maternas y fetales es necesario que la atención esté a cargo de un equipo interdisciplinario (diabetólogo, obstetra, neonatólogo, educador, Lic. en Nutrición, entre otros).

\section{Frecuencia de visitas}

Se recomienda:

- A partir del diagnóstico realizar visitas semanales hasta lograr metas de control metabólico.

- Posteriormente realizar visitas cada 15 días hasta la semana 32, y luego semanales hasta la internación, siempre que no se presente ninguna patología concomitante o complicación que requiera controles más seguidos.

- En el seguimiento posterior a una internación durante el embarazo, la citación debe hacerse durante los siete días siguientes al alta hospitalaria.

\section{Evaluación clínica}

Se aconseja:

- Evaluar las siguientes condiciones socio-demográficas: ingreso a control prenatal después de las 20 semanas de gestación, edad menor de 17 años o mayor de 35 años, desocupación personal y/o familiar, analfabetismo, desnutrición, obesidad y violencia doméstica.
- Realizar anamnesis alimentaria con el fin de evaluar y ajustar el plan alimentario a la nueva situación biológica.

- Incluir el examen clínico habitual con evaluación del estado nutricional.

- En las visitas posteriores repetir examen físico completo y registro de peso, evaluar la presencia de edema en los miembros inferiores, tensión arterial y el control metabólico mediante los automonitoreos glucémicos y el control de la cetonuria cuando se considere necesario.

\section{Control nutricional}

La ganancia óptima de peso durante la gestación depende del estado nutricional previo determinado por el IMC.

Se recomienda:

- Contemplar una ganancia de peso según el estado nutricional previo al embarazo como se indica en la Figura 1 y Tabla 1.

- No se considera conveniente el descenso de peso durante el embarazo en las pacientes obesas.

- En mujeres con estado nutricional normal previo al embarazo, que la ganancia de peso estimada durante la gestación sea de 400 a 500 g por semana a partir de la segunda mitad del embarazo (20 semanas).

- Si la ganancia de peso es mayor a la esperada, descartar edemas, sedentarismo, ingesta con elevado valor calórico total y polihidramnios.

- Si la ganancia de peso es sub-óptima, evaluar restricción calórica que complique el crecimiento y desarrollo del feto, aumento de actividad física, vómitos, diarrea o subalimentación por parte de la paciente.

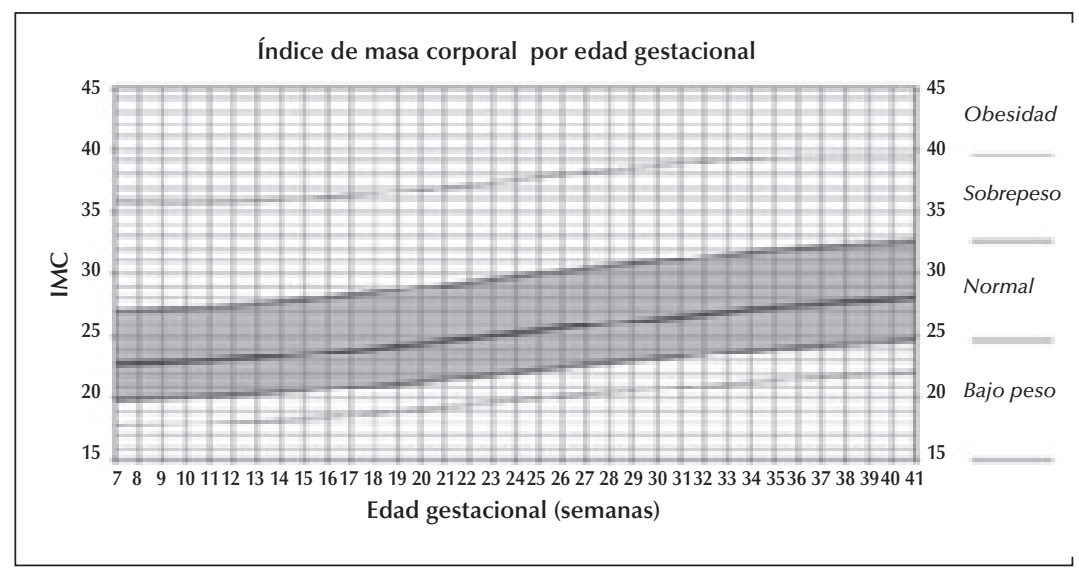

Figura 1: Incremento de peso según IMC. Calvo y col. 


\begin{tabular}{|l|l|}
\hline $\begin{array}{c}\text { Estado nutricional previo } \\
\text { (según IMC) }\end{array}$ & \multicolumn{1}{|c|}{$\begin{array}{c}\text { Ganancia de peso recomendada en } \\
\text { todo el embarazo en } \mathbf{~ k g}\end{array}$} \\
\hline Bajo peso (menor a $18,5 \mathrm{~kg})$ & 12,5 a $18 \mathrm{~kg}$ \\
\hline Peso normal $(18,5-24,9 \mathrm{~kg})$ & 11 a $16 \mathrm{~kg}$ \\
\hline Sobrepeso $(25-29,9 \mathrm{~kg})$ & 7,0 a $11 \mathrm{~kg}$ \\
\hline Obesidad (>30) & 5 a $7 \mathrm{~kg}$ \\
\hline
\end{tabular}

Fuente: Adaptado de Rasmussen et al.

Tabla 1: Ganancia de peso adecuada durante el embarazo.

\section{Control metabólico}

El control metabólico se establece con la evaluación del peso y el automonitoreo glucémico. Puede complementarse con las determinaciones de fructosamina y $\mathrm{HbA} 1 \mathrm{c}$ en laboratorio.

El control metabólico se considera óptimo con:

- Glucemia en ayunas entre 70 y 90 mg/dl.

- Glucemia 1 hora postprandial entre 90 y $140 \mathrm{mg} / \mathrm{dl}$

- Glucemia 2 horas postprandial entre 90 y $120 \mathrm{mg} / \mathrm{dl}$.

- Evitar hipoglucemias o glucemias $<60$ mg/dl.

- Ganancia de peso adecuada.

- HbA1c: tener en cuenta que se modifica con la vida media del eritrocito y ésta disminuye durante el embarazo normal debido a mayor hematopoyesis en respuesta a los niveles de eritropoyetina elevados; también se modifica con la anemia.

- Fructosamina: si bien los valores de corte propuestos son menores a 260 umol/l, no hay suficiente evidencia de valores de corte en el embarazo.

En conjunto, los parámetros de control considerados de referencia son: los valores del monitoreo glucémico y la ausencia de hipoglucemias.

\section{Recomendaciones}

- Efectuar un seguimiento de peso con la curva de incremento de peso para embarazadas según IMC o curvas de Calvo.

- Lograr la obtención de un óptimo control metabólico que permita asemejar los niveles glucémicos a los de las embarazadas no diabéticas a fin de evitar las complicaciones feto-neonatales.

Fuerza de las recomendaciones: alta. Calidad de la evidencia: fuerte a favor.

\section{Frecuencia de automonitoreos}

Control de glucemia:

- Se recomiendan entre tres a siete monitoreos diarios los cuales podrán ser pre y/o postprandiales (y en la madrugada cuando sea necesario) de acuerdo al tratamiento adoptado.

- Adecuarlo a las necesidades propias de cada paciente, al criterio médico y a la disponibilidad de insumos.

\section{Laboratorio}

Se recomienda:

- En la primera consulta solicitar: hemograma, glucemia, uricemia, colesterolemia, trigliceridemia, HbA1c o fructosamina y orina completa.

- Pedir un urocultivo en cada trimestre.

- Solicitar las serologías para sífilis, toxoplasmosis, chagas, hepatitis B y VIH, con la misma frecuencia que en la embarazada no diabética.

- En caso de embarazo no programado dosar $\mathrm{TSH}$, T4 libre para monitoreo de tratamiento en caso de trastornos tiroideos previos y considerar anticuerpos antitiroperoxidada tiroidea.

- Evaluar la función renal: urea, creatinina y proteinuria de 24 hs en cada trimestre o según criterio médico.

\section{Control oftalmológico}

En pacientes con diabetes pregestacional solicitar examen de retina en cada trimestre. En caso de hallar alguna alteración realizar el seguimiento y tratamiento indicado por el oftalmólogo.

\section{Control odontológico}

Lograr el control de la salud bucal mediante la derivación al odontólogo a partir de la primera consulta con el médico. En caso de presentar alguna alteración, el odontólogo decidirá la periodicidad de las consultas.

\section{Vacunación durante el embarazo}

La importancia de la vacunación durante el embarazo radica no sólo en la protección de las mujeres vacunadas, sino también de forma indirecta del recién nacido durante los primeros meses de vida.

- En el embarazo se recomiendan las vacunas influenza (aplicada durante época estival, en cualquier momento de la gestación) y DTPa (difteria, tos convulsa, tétanos, acelular) luego de la semana 20 de gestación.

- Evaluar requerimientos de otras vacunas (he- 
patitis $A$, hepatitis $B$, neumococco y/o meningococco) en circunstancias particulares (presencia de factores de riesgo o alta exposición).

\section{2) Seguimiento obstétrico}

El control prenatal (CPN) en mujeres con diabetes es un desafío para el trabajo en equipo. Es un período de absoluta vulnerabilidad para las mujeres y sus familias, por ello, el empoderamiento y la información adecuada, basada en evidencia y centrada en la familia, así como también el respeto a los derechos juegan un rol primordial. Se recomienda realizar el CPN según lo indicado en la Tabla 2.

\begin{tabular}{|c|l|}
\hline $\begin{array}{c}\text { Edad } \\
\text { gestacional }\end{array}$ & \multicolumn{1}{|c|}{ Cuidado } \\
\hline $7-0$ semanas & Confirmar viabilidad y edad gestacional \\
\hline 10 semanas & $\begin{array}{l}\text { - Explicar vía de finalización del embarazo } \\
\text { - Recomendar e informar acerca de los beneficios de } \\
\text { la lactancia }\end{array}$ \\
\hline 20 semanas & $\begin{array}{l}\text { - Ecocardiograma fetal } \\
\text { - Ecografía de } 2^{\circ} \text { nivel }\end{array}$ \\
\hline 28 semanas & $\begin{array}{l}\text { Ecografía para control de crecimiento y test del líquido } \\
\text { amniótico (ILA) }\end{array}$ \\
\hline 32 semanas & $\begin{array}{l}\text { - Ecografía } \\
\text { - Monitoreo fetal }\end{array}$ \\
\hline 36 semanas & $\begin{array}{l}\text { • Monitoreo fetal } \\
\text { nejo inicial del recién nacido y lactancia, anticoncepción }\end{array}$ \\
\hline$>36$ semanas & Evaluar momento y vía de finalización del embarazo \\
\hline
\end{tabular}

Tabla 2: Control obstétrico prenatal en mujeres con diabetes.

\section{Finalización del embarazo}

\section{Momento de finalización}

La finalización electiva del embarazo a término no ha demostrado disminuir la tasa de cesáreas, macrosomía y complicaciones intraparto. Una revisión sistemática de Cochrane no encontró evidencia de la utilidad de finalización electiva en pacientes con buen control glucémico. El manejo expectante resultó ser costo-efectivo.

\section{Considerar en la gestante con DM1 y DM2:}

- Finalización antes de semana 37 si existen complicaciones metabólicas, maternas o fetales.

- Se sugiere ofrecer inducción al trabajo de parto en semana 37-38,6 si las condiciones obstétricas lo permiten.

- El American Congress of Obstetricians and
Gynecologists (ACOG) sugiere en DM pregestacional sin vasculopatía finalización a partir de la semana 39 y en DM pregestacional con vasculopatía finalización en la semana 37-39.

- La prolongación del embarazo más allá de la semana 40 en pacientes diabéticas se asoció a un aumento de la morbimortalidad fetal, por lo que se recomienda el nacimiento antes de completar las 40,6 semanas de gestación.

Se finalizará electivamente el embarazo sólo ante las siguientes situaciones:

- Pobre regulación metabólica.

- Compromiso de salud fetal.

- Score de Bishop >6 con más de 37 semanas cumplidas.

- Patología obstétrica que requiera interrupción del embarazo.

\section{Vía de finalización}

El parto vaginal de un bebé macrosómico en el contexto de una paciente con diabetes se asocia a un riesgo aumentado de trauma intraparto.

Se recomienda efectuar una estimación del peso fetal por ecografía alrededor de la semana 38 a los fines conocer aquellas mujeres con fetos con cálculo de peso fetal $>4.500 \mathrm{~g}$ en quienes, por su mayor probabilidad de sufrir complicaciones intraparto, debe programarse una cesárea en la semana 39. En aquellas con cálculo de peso fetal entre 4.000 a $4.499 \mathrm{~g}$ considerar historia obstétrica, polimetría y progreso del trabajo de parto.

Tener en cuenta que la estimación del peso fetal por ultrasonografía presenta una variación muy amplia; esta dispersión ha sido estimada al término en $\pm 20 \%$. Aún así es junto con la clínica (altura uterina y Leopold) una estrategia de abordaje útil para la estimación de peso fetal.

\section{Recomendaciones}

- Se recomienda enfáticamente la necesidad de consensuar todas las decisiones sobre la finalización del embarazo, con detalle de riesgos y beneficios con las madres y sus familias.

- Es ideal esperar el inicio espontáneo del trabajo de parto; no hay evidencia que avale la necesidad de finalización temprana del embarazo en mujeres con buen control metabólico.

- La diabetes mellitus no debería considerarse per se una contraindicación para parto vaginal después de la cesárea. 


\section{Tratamiento}

\section{Plan de alimentación}

- El objetivo del plan nutricional durante el embarazo que cursa con diabetes es mejorar o mantener un adecuado estado nutricional, optimizar el control glucémico y lipídico, evitar la hiperglucemia materna preprandial y reducir las excursiones de la glucemia postprandial.

- Se debe garantizar a la mujer un ingreso suficiente de energía y nutrientes para permitir el crecimiento normal del feto y evitar un excesivo aumento de peso y el impacto en el feto de la macrosomía.

- Los consejos nutricionales deben enmarcarse en un programa de educación terapéutica sistemático adaptados a la realidad socioeconómica de las mujeres con diabetes.

- Se recomiendan aproximadamente $30 \mathrm{kcal} / \mathrm{pes}$ teórico de la paciente y sumar $300 \mathrm{Kcal}$ más a partir del segundo trimestre. En caso de adolescentes y embarazo múltiple sumar $450 \mathrm{kcal}$ desde el primer trimestre. El valor calórico total (VCT) no debe ser inferior a $1.700 \mathrm{kcal} /$ día.

- Las recomendaciones para macronutrientes son 50 a $60 \%$ de las calorías diarias de carbohidratos ( $\mathrm{CH}$, complejo, alto contenido de fibra), 10 a $20 \%$ de proteína, y 25 a $30 \%$ de grasa (menos del $10 \%$ saturada).

- Deben ofrecerse alimentos de bajo índice glucémico (IG) (menos de 55).

- Alimentos con bajo IG: granos enteros, frutas, verduras.

- Se sugiere que el contenido de $\mathrm{CH}$ en cada comida sea:

- Desayuno: 15 a $45 \mathrm{~g}$.

- Almuerzo y cena 45 a 75 g en cada comida.

- Colaciones: 15 a $45 \mathrm{~g} \mathrm{CH}$.

- Deben evaluarse los niveles de glucemia en ayunas para ofrecer una colación antes de acostarse. La colación debe incluir una o dos porciones de carbohidratos ricos en fibra con una o dos porciones de proteína (por ejemplo, una o dos rebanadas de pan y una o dos fetas de queso).

- Considerar la dieta recomendada para gestantes con diabetes (Tabla 3), recordando que la dieta debe brindarse en forma personalizada.

\begin{tabular}{|l|l|}
\hline $\begin{array}{l}\text { Recomendaciones diarias } \\
\text { permitidas de carbohidratos }\end{array}$ & $\begin{array}{l}\text { Un mínimo de } 175 \mathrm{~g} / \text { día en carbohidratos preferiblemente complejos. Las porciones deben } \\
\text { distribuirse entre las tres comidas y dos o tres colaciones para minimizar la hiperglucemia post- } \\
\text { prandial. Limitar la fruta a dos porciones por día y evitar su consumo en el desayuno o la noche }\end{array}$ \\
\hline $\begin{array}{l}\text { Recomendaciones diarias } \\
\text { permitidas de proteínas }\end{array}$ & En embarazo único $1 \mathrm{~g} / \mathrm{Kg}$ peso teórico y sumar $25 \mathrm{~g}$ por día y 50 g por día si es múltiple \\
\hline Grasas & $\begin{array}{l}\text { El } 20-35 \% \text { del total de energía. Incorporar grasas insaturadas, valores mínimos de ácido lino- } \\
\text { leico }(13 \mathrm{~g}) \text { y ácido alfa linolénico }(1,4 \mathrm{~g}) \text { cuyas fuentes son aceite de soja, canola, maíz y/o } \\
\text { girasol. Grasas saturadas }<10 \% \text {. Evitar grasas trans }\end{array}$ \\
\hline Edulcorantes artificiales & $\begin{array}{l}\text { Aspartame, acesulfame y sucralosa se permiten con moderación durante el embarazo con tres a } \\
\text { cinco porciones por día }\end{array}$ \\
\hline Fibra & Evitar su consumo \\
\hline Cafeína & No más de $300 \mathrm{mg} /$ día $(\sim 2$ tazas). \\
\hline
\end{tabular}

Tabla 3: Dieta recomendada en gestantes con diabetes.

\section{Considerar que:}

- Se aconseja que el desayuno sea pequeño con no más de dos porciones de hidratos de carbonos y una o dos porciones de proteínas para controlar el aumento de la resistencia a la insulina en la mañana y evitar la hipoglucemia postprandial. Evitar la fruta en el desayuno.
- Se recomienda leche entera para mejorar la absorción de la vitamina $\mathrm{D}$ y el calcio.

- El embarazo es una situación fisiológica perdedora de sodio, por lo cual se recomienda no menos de $5 \mathrm{~g}$ de cloruro de sodio/día. Debe estar mínimamente restringido en hipertensión arterial sensible a la sal y en insuficiencia cardíaca. 


\section{Insulinoterapia}

\section{Recomendaciones}

La insulina es el tratamiento de elección en pacientes con diabetes pregestacional ya sea en las mujeres con DM1 ó DM2 tratadas previamente con dieta con o sin antidiabéticos orales.

Fuerza de las recomendaciones: alta. Calidad de la evidencia: fuerte a favor.

El esquema de insulinoterapia es artesanal y personalizado para cada paciente

Pueden considerarse situaciones comunes a tener en cuenta como:

- En la DM pregestacional tipo 1 el nivel de glucemia en ayunas es menor y el aumento postprandial es relativamente mayor que en la mujer no embarazada.

- La excursión postprandial vuelve a la línea basal más rápidamente, particularmente a medida que el embarazo progresa.

- En el primer trimestre hay una disminución en los requerimientos diarios de insulina, y las mujeres, especialmente aquellas con DM1, pueden experimentar un aumento en los episodios de hipoglucemia.

- A partir del segundo trimestre aumenta la resistencia a la insulina, lo cual habitualmente produce un aumento en el requerimiento de insulina con necesidad de ajustes semanales de la dosis para alcanzar los objetivos glucémicos. Por ello, en general, una proporción más pequeña de la dosis diaria total se administra como insulina basal (menos del $50 \%$ ) y una proporción mayor (más del $50 \%$ ) como insulina prandial.

- Al final del tercer trimestre puede producirse una pequeña y gradual disminución de los requerimientos de insulina.

\section{Análogos de insulina en el embarazo}

- El análogo ultrarrápido de insulina aspártica, aprobado por la ANMAT (categoría B), demostró (Mathiesen et al. 2007) menor excursión en las glucemias postprandiales y un menor riesgo de hipoglucemia severa en mujeres con DM1.

- La insulina lispro (categoría B para la Food and Drug Administration, FDA) no está aprobada por la ANMAT en Argentina por falta de estudios adecuados y controlados en el embarazo.
- No existen datos publicados de estudios aleatorizados con el uso del análogo ultrarrápido glulisina en el embarazo (categoría C).

- El análogo lento de insulina detemir fue aprobado para el uso en el embarazo (categoría B para FDA) y demostró (Hod et al. 2014) una tendencia a menor glucemia de ayuno con resultados comparables en $\mathrm{HbA} 1 \mathrm{c}$ e incidencia de hipoglucemias comparado con insulina NPH.

- Los análogos de insulina glargina y degludec no están aprobados por las entidades regulatorias por falta de estudios durante el embarazo (categoría C).

\section{Consideraciones del esquema de insulinización en el embarazo}

- Si son pacientes previamente insulinizadas se recomienda mantener el esquema (según insulinas aprobadas para su uso en el embarazo) con ajustes correspondientes e intensificando con esquema basal-bolo.

- En pacientes con DM2 previamente tratadas con dieta o agentes orales, de tener que comenzar con insulina, se recomienda comenzar con 0,2 Ul/ $/ \mathrm{kg}$ peso día de insulina NPH o análogo detemir con el objetivo de lograr glucemias de ayuno adecuadas y eventualmente agregar insulina rápida o análogo ultrarrápido (aspártica) si no logran controlarse los perfiles postprandiales.

\section{Recomendaciones}

Como primera elección utilizar insulina humana:

- Insulina basal: insulina de acción intermedia NPH (categoría B, FDA) como insulina basal o análogo lento detemir (categoría B, FDA).

- Insulina preprandial (bolo): insulina de acción rápida corriente o regular humana (categoría A) (categoría B) o análogo de acción rápida aspártica (categoría B, FDA), por ser la única aprobada por la ANMAT en la actualidad.

El esquema de insulinoterapia es personalizado y optimizado con el objetivo de cumplir con los criterios del buen control metabólico. En cada país deben respetarse los lineamientos de las entidades regulatorias correspondientes.

Fuerza de las recomendaciones: alta. Calidad de la evidencia: fuerte a favor.

\section{Hipoglucemiantes orales}

No están avalados por las entidades regulatorias para su utilización en el embarazo. 
Considerar que:

- La metformina pertenece a la categoría B para FDA en el embarazo. Diversos estudios demuestran que la metformina no se asoció a un aumento de las complicaciones perinatales, excepto un incremento de embarazo pretérmino.

- La glibenclamida pertenece a la categoría B para FDA en el embarazo. Su administración se asoció con mayor peso al nacer en el recién nacido, mayor incidencia de hipoglucemia neonatal y macrosomía comparada con insulina. Además se relacionó con una mayor ganancia de peso materno, mayor peso al nacer en el recién nacido, mayor incidencia de hipoglucemia neonatal y de macrosomía que la metformina.

- Los agentes orales atraviesan la placenta. Ninguno de estos estudios o metaanálisis evaluaron los resultados de sus efectos a largo plazo en la descendencia. Si bien no se han demostrado efectos adversos sobre el feto, faltan estudios a largo plazo que avalen su utilización.

\section{Tecnología aplicada al tratamiento de la diabetes en el embarazo}

\section{Infusores de insulina}

Los infusores de insulina brindan mayor flexibilidad y un ajuste de dosis más preciso al mejorar el control glucémico, y reducir la variabilidad y las hipoglucemias.

Sin embargo, estudios controlados randomizados no demostraron ventajas o desventajas del uso de infusores de insulina sobre las múltiples dosis en el embarazo en términos de control glucémico, complicaciones maternas o fetales. Por otro lado, el costo-efectividad de estas tecnologías y la capacidad de los sistemas de salud para afrontarlas requieren un cuidadoso análisis y mejor evidencia.

Considerar:

- Enseñar a rotar sitios de aplicación del set de infusión, dado que el tejido subcutáneo abdominal se adelgaza para obtener patrones de absorción más predecibles.

- Continuar con la infusión subcutánea de insulina durante el parto que resultaría seguro y eficaz.

\section{Recomendaciones}

- Continuar con el uso del infusor de insulina en pacientes que lo utilizaban antes del embarazo.

- Podrá iniciarse tratamiento con infusor de insulina en etapa preconcepcional si no se alcanzan los objetivos glucémicos ( $\mathrm{HbAc}>8,5 \%$ o hipoglucemias severas o no percibidas).

Fuerza de las recomendaciones: moderada. Calidad de la evidencia: fuerte a favor de las intervenciones indicadas.

No se recomienda iniciar terapia con infusor durante el embarazo a menos que otras estrategias hayan sido probadas y no resultaran exitosas.

Fuerza de la recomendación: moderada. Calidad de la evidencia: fuerte en contra de la intervención indicada.

\section{Monitoreo continuo de glucosa}

El monitoreo continuo de glucosa podría indicarse cuando el automonitoreo glucémico capilar, la HbA1c y la fructosamina no son suficientes para evaluar el control metabólico. Es útil para identificar períodos de hipo o hiperglucemia y establecer la variabilidad glucémica.

Se sugiere emplear en las siguientes situaciones:

- Hipoglucemia severa, con o sin deterioro de la percepción.

- Niveles glucémicos inestables.

- Para obtener información sobre variabilidad.

- No ofrecer rutinariamente el monitoreo continuo de glucosa. Los sistemas disponibles actualmente no son tan precisos en niveles glucémicos menores de $70 \mathrm{mg} / \mathrm{dl}$ y sus beneficios en la evolución del embarazo aún no son claros con las evidencias disponibles.

- Es fundamental la atención por parte de un equipo con experiencia en el uso del monitoreo continuo de glucosa.

La tecnología ha evolucionado significativamente y se necesitarán estudios con las nuevas generaciones de infusores (con o sin monitoreo continuo de glucosa) para establecer su rol en el tratamiento de la diabetes durante el embarazo, evaluar su efectividad y aceptabilidad. 


\section{Actividad física}

La intervención con ejercicio físico en diabetes pregestacional tiene como objetivo mejorar el control glucémico. El ejercicio ayuda a controlar la excesiva ganancia de peso, los parámetros glucémicos y lipídicos. Al mejorar la sensibilidad a la insulina, asegura sensación de bienestar y es útil para reforzar la adherencia al plan de alimentación y el tratamiento insulínico.

\section{Considerar:}

- En las mujeres que inicien un programa de ejercicio aumentar la duración y la intensidad de éste en forma gradual, además de adaptar la dieta y la dosis de insulina en forma personalizada y según monitoreo glucémico.

- Realizar monitoreo glucémico antes, durante y luego de realizar actividad física para evitar hipoglucemias.

- Debe ajustarse la terapia nutricional en relación a la actividad física acordada. Dicha actividad debe establecerse en forma individual mediante prescripción a fin de incrementar la adhesión al programa de ejercicios.

Se recomienda:

- En ausencia de contraindicaciones estimular a la gestante a realizar ejercicio aeróbico de intensidad leve a moderada en forma regular, en períodos de 30-45 minutos al menos tres veces por semana.

- Evaluar la relación riesgo-beneficio que el ejercicio supone para la madre y el feto.

El ejercicio físico aeróbico está contraindicado durante la gestación de pacientes con diabetes pregestacional que presentan hipertensión arterial preexistente, preeclampsia, embarazos múltiples, retinopatía proliferativa, nefropatía, neuropatía periférica, coronariopatía, disfunción autonómica, ausencia de mecanismos contrarreguladores de hipoglucemia, hipoglucemia o hiperglucemia con cetosis, sangrado uterino, cérvix incompetente y si aumentan las contracciones uterinas.

\section{3) Complicaciones agudas}

\section{Hipoglucemia}

Durante el embarazo ocurren importantes cambios en el metabolismo materno, el cual se adapta para asegurar un adecuado aporte de nutrientes a la madre y a la unidad feto-placentaria.

\section{Considerar que:}

- En pacientes DM1 la hipoglucemia severa (hipoglucemia que requiere de la ayuda de terceras personas para su recuperación) es entre tres a cinco veces mayor en el primer trimestre que previo al embarazo.

- Los principales factores de riesgo para presentar hipoglucemia grave durante el primer trimestre del embarazo son la historia de hipoglucemia grave previa, hipoglucemias desapercibidas, duración de la diabetes de 10 años o más, $\mathrm{HbA} 1 \mathrm{c}<6,5 \%$, gastroparesia y dosis muy elevadas de insulina.

- La glucemia en ayunas promedio durante el embarazo es de $75 \pm 12 \mathrm{mg} / \mathrm{dl}$ por lo cual valores inferiores a la media más 1 DS $(63 \mathrm{mg} / \mathrm{dl})$ corresponderían a hipoglucemia. El mantenimiento prolongado de concentraciones de glucosa reducido durante el embarazo se asocia a un aumento del riesgo de tener un recién nacido de bajo peso para su edad gestacional.

- Tratar todos los episodios de hipoglucemia, incluso en ausencia de síntomas, para evitar que se ponga en marcha la liberación de hormonas de contrarregulación y para asegurar un flujo de glucosa acorde a las necesidades del crecimiento fetal. Es necesario que el entorno de la paciente sepa reconocer los síntomas de hipoglucemia para ayudarla si tiene una hipoglucemia severa (pérdida de conocimiento) y esté instruido en el uso del glucagón. El glucagón se coloca en forma subcutánea en dosis de 1 mg y la glucemia se monitorea hasta que la paciente sea capaz de ingerir. El glucagón no pasa a la placenta y su uso en el embarazo demostró no tener riesgos.

- Una vez que se efectuó el tratamiento adecuado y los valores de glucemias se normalizaron debe evaluarse el motivo de esa hipoglucemia para determinar si la paciente está en riesgo de un nuevo episodio en las próximas horas, analizar la necesidad de modificaciones en el tratamiento o identificar eventuales errores en la autoadministración de insulina en relación al automonitoreo, la ingesta y la actividad física e infecciones, etc. y así evitar la aparición de nuevos episodios. 


\section{Recomendaciones}

- Educar e informar a todas las mujeres que planean el embarazo o ya embarazadas acerca del reconocimiento y el tratamiento de la hipoglucemia.

- Tratar la hipoglucemia inmediatamente al ser advertida con $15 \mathrm{~g}$ de hidratos de carbono de acción rápida y determinar la glucemia capilar a los 15 minutos de la ingesta. Si la glucemia es menor a $80 \mathrm{mg} / \mathrm{dl}$ o los síntomas no se resuelven administrar $15 \mathrm{~g}$ de hidratos de carbono adicionales.

- Para evitar hipoglucemias repetidas emplear el análogo aspártica dado que demostró disminuir la frecuencia de hipoglucemias graves y no graves en comparación con la insulina regular.

- Si bien no está indicado el monitoreo continuo en tiempo real, considerar la posibilidad de su uso cuando hay hipoglucemias severas, siempre y cuando exista un equipo de soporte con experiencia en su empleo.

Fuerza de la recomendación: moderada. Calidad de la evidencia: fuerte en contra de la intervención indicada.

No tratar con carbohidratos en forma irrestricta hasta que los síntomas mejoren porque frecuentemente se genera hiperglucemia posterior.

Fuerza de la recomendación: moderada. Calidad de la evidencia: fuerte en contra del tratamiento indicado.

\section{Cetoacidosis}

La cetoacidosis durante el embarazo es una de las mayores causas de mortalidad perinatal.

Considerar que:

- Se requiere un alto índice de sospecha dado que la aparición de cetoacidosis diabética durante el embarazo puede ser insidiosa; por lo general se presenta con niveles más bajos de glucosa que fuera del mismo y, a menudo, progresa más rápidamente en comparación con diabéticas no embarazadas.

- En el embarazo el $10-30 \%$ de los casos de cetoacidosis se producen con los niveles de glucosa moderadamente elevados (<250 mg/dl).

- Aunque la cetoacidosis generalmente se ob- serva en pacientes con DM1, también puede ocurrir en mujeres con DM2.

- Las causas más frecuentes para la aparición de cetoacidosis incluyen: infección, omisión de dosis de insulina, dosis insuficiente de insulina y uso de fármacos beta-simpaticomiméticos y glucocorticoides.

- El enfoque terapéutico es similar al de las pacientes no embarazadas y supone la hospitalización inmediata y la terapia dirigida con vigilancia intensiva (insulina, hidratación, potasio y manejo de la causa desencadenante).

\section{Recomendaciones}

- Educar a todas las mujeres con diabetes preexistente que planean el embarazo o ya están embarazadas acerca de la cetoacidosis.

- Enseñar a la paciente a realizar mediciones de cetonas en la orina en momentos de enfermedad o cuando presenta niveles de glucosa superiores a $200 \mathrm{mg} / \mathrm{dl}$ persistentes.

- Monitorear la frecuencia cardíaca fetal continua y realizar pruebas biofísicas para evaluar el bienestar fetal en los casos que ocurren después de las 24 semanas de gestación.

Fuerza de las recomendaciones: moderada. Calidad de la evidencia: fuerte a favor.

\section{4) Situaciones especiales}

\section{Hipertensión arterial}

La diabetes pregestacional incrementa la frecuencia de los estados hipertensivos del embarazo, patología que aumenta el riesgo de morbilidad y mortalidad materna y perinatal. Además de la diabetes preexistente, otros factores de riesgo para preeclampsia son el mal control metabólico, la obesidad, la nuliparidad, la preeclampsia en embarazos anteriores y los antecedentes familiares de preeclampsia (madre o hermana).

\section{Considerar que:}

- La preeclampsia complica hasta el $30 \%$ de los embarazos en mujeres con diabetes.

- La preeclampsia es más frecuente en mujeres con DM1, mientras que la hipertensión crónica es más frecuente en aquellas con DM2. La preeclampsia sobreimpuesta a la hipertensión crónica es la situación de mayor morbimortaldad materna. 


\section{Recomendación}

- Diagnosticar hipertensión en el embarazo o estado hipertensivo del embarazo cuando la prensión arterial sistólica es igual o mayor a 140 mm Hg y/o la presión arterial diastólica es igual o mayor a $90 \mathrm{~mm} \mathrm{Hg}$, en dos tomas separadas por lo menos por 6 hs.

- Derivar a un servicio de urgencia para descartar preeclampsia y realizar evaluación integral con laboratorio (hemograma con plaquetas, hepatograma, creatinina, KPTT, Quick, LDH, ácido úrico) y control de vitalidad fetal con ecografía y doppler.

Fuerza de la recomendación: alta. Calidad de la evidencia: fuerte a favor.

\section{En la gestante con hipertensión}

Se recomienda:

- Evaluación clínica mediante una anamnesis orientada a la patología y examen clínico completo.

- Exámenes de laboratorio: solicitar hemograma completo, creatinina plasmática, proteinuria de 24 hs, uricemia, hepatograma, estudio de coagulación y LDH.

- Ecodoppler fetal en embarazos de más de 26 semanas.

- Fondo de ojo en hipertensión crónica.

- Evaluación cardiovascular.

- La tomografía computarizada (TAC) y la resonancia magnética (RM) se reservarán sólo para los casos con manifestaciones de complicación neurológica o ceguera cortical.

Es importante ajustar la periodicidad y la frecuencia de la realización de estos estudios a cada caso según la evolución clínica y los resultados de laboratorio previos.

\section{Considerar que:}

- Tratar la hipertensión leve o moderada puede reducir el riesgo de desarrollar hipertensión grave, aunque no se ha demostrado prevención de la preeclampsia ni modificación de las alteraciones fisiopatológicas de la misma.

- Ante el tratamiento antihipertensivo, el descenso brusco o severo de la presión arterial disminuye el flujo útero-placentario y podría producir alteraciones en la salud fetal.
- Cuando se indica tratamiento farmacológico las metas son prevenir la eclampsia, las complicaciones severas cerebrovasculares, cardiovasculares y la muerte materna.

- La finalización del embarazo es la terapia apropiada para la madre, pero puede no serlo para el feto y/o neonato.

Se aconseja:

- No reducir la presión arterial sistólica a menos de 120 mm Hg y la presión arterial diastólica a menos de $80 \mathrm{~mm} \mathrm{Hg}$. El nivel promedio de la presión arterial en el embarazo es $116 \pm 12$ de sistólica y $70 \pm 7$ de diastólica.

\section{Tratamiento no farmacológico}

Se recomienda:

- Reducir la actividad física y la jornada laboral pero no sugerir el reposo en cama prolongado porque aumenta el riesgo de tromboembolismo.

- No restringir el sodio en la dieta de pacientes con hipertensión gestacional o preeclampsia. Sólo corresponde continuar dieta hiposódica en hipertensas crónicas.

- Contraindicar el consumo de alcohol y tabaco. Consumir alcohol puede empeorar la hipertensión materna. Evitar que la embarazada sea una fumadora pasiva. El hábito de fumar se asocia a desprendimiento de placenta y restricción del crecimiento intrauterino (RCIU).

\section{Tratamiento farmacológico}

Recomendaciones para la prevención de la preeclampsia:

- Prácticas recomendadas:

- Administración de suplemento de calcio durante el embarazo en áreas donde el consumo de calcio es bajo (<900 mg/día).

- Administración de ácido acetil salicílico en dosis bajas (aspirina $75 \mathrm{mg}$ ) en las mujeres que tienen un alto riesgo de desarrollar la enfermedad.

- Prácticas no recomendadas:

- Administración de suplemento de vitamina D.

- Administración de suplemento de calcio durante el embarazo en áreas donde no hay deficiencia en el consumo de calcio.

- Administración de suplemento de vitaminas C y E como monoterapia o combinados.

- Uso de diuréticos, particularmente tiazídicos. 


\section{Recomendaciones}

Las drogas para el tratamiento antihipertensivo por vía oral son:

- Alfametildopa: 500 a 2.000 mg/día

- Labetalol: 200 a 1.200 mg/día

- Nifedipina: 10 a 40 mg/día. Se prefieren los preparados de liberación lenta

Fuerza de las recomendaciones: alta. Calidad de la evidencia: fuerte a favor de la intervención con estas drogas.

Las drogas contraindicadas en el embarazo son:

- Inhibidores de enzima convertidora (IECA)

- Antagonistas de angiotensina II (ARA II)

- Prazosin

- Atenolol

Prazosin y atenolol no se recomiendan en el embarazo por asociarse a retardo del crecimiento intrauterino, oligoamnios y muerte fetal. El prazosin también se asocia a complicaciones renales del recién nacido.

Fuerza de las recomendaciones: alta. Calidad de la evidencia: fuerte en contra de intervenir con estas drogas.

\section{Nefropatía}

Se debe tener en cuenta que:

- Los cambios fisiológicos del embarazo incluyen modificaciones en la hemodinamia renal, con hiperfiltración glomerular. Estos cambios se normalizan entre seis a ocho semanas después del embarazo.

- La creatininemia disminuye en el embarazo. Se consideran normales valores menores a 0,9 mg/dl; es decir que valores considerados normales en mujeres no embarazadas pueden corresponder a un importante deterioro de la función renal durante la gestación.

- Las pacientes con insuficiencia renal severa (creatininemia mayor o igual a $1,5 \mathrm{mg} / \mathrm{dl}$ ) tienen entre un 30 y $50 \%$ de riesgo de empeorar su función renal.

- La proteinuria se incrementa durante el embarazo en gestantes con nefropatía diabética. Las que tienen filtrado glomerular normal difícilmente queden con deterioro de la función renal permanente si se compensa metabólicamente y se mantiene normotensa.

- Los factores que contribuyen a empeorar la nefropatía en el embarazo son el aumento de la in- gesta proteica, incremento de la ingesta de sodio, ejercicio intenso, infecciones urinarias, hipertensión y suspensión de los "priles" o los "sartanes".

- El diagnóstico de nefropatía diabética se realiza ante la presencia de albuminuria persistente, mayor de 300 mg/día en las primeras 20 semanas de embarazo, en ausencia de infección urinaria.

- Cuando se identifica nefropatía diabética después de la semana 20 de gestación hay que descartar preeclampsia. El comienzo brusco de proteinuria con empeoramiento de la hipertensión en gestantes con hipertensión crónica sugiere preclampsia sobreimpuesta.

- El embarazo per se no empeora la nefropatía diabética en pacientes con creatinina normal, pero puede acompañarse de complicaciones como restricción del crecimiento fetal intrauterino, preeclampsia y parto pretérmino. Con gran frecuencia se requiere interrumpir el embarazo antes de la 36 semana.

\section{Recomendaciones}

- Programar el embarazo en pacientes con nefropatía para establecer el riesgo para la madre y el feto.

- Realizar en estas pacientes un estricto seguimiento y control metabólico y de la presión arterial durante el embarazo.

Fuerza de la recomendación: alta. Calidad de la evidencia: fuerte a favor.

\section{Retinopatía}

Considerar que:

- Estudios en pacientes con DM1 y DM2 demostraron que el embarazo puede acelerar la progresión de la retinopatía diabética. Sin embargo, no produce empeoramiento definitivo en las retinopatías no proliferativas; el mayor riesgo se da en las retinopatías proliferativas severas, hipertensión crónica, preeclampsia y mal control glucémico previo al embarazo.

- Los factores de riesgo más frecuentes para el deterioro de la retinopatía durante el embarazo son: duración de la diabetes $>10$ años, retinopatía moderada o severa y descenso brusco de la $\mathrm{HbA} 1 \mathrm{c}$ y reiteradas hipoglucemias moderadas a severas.

- Se considera que la retinopatía empeora cuando pasa a un estadio posterior y/o desarrolla edema de mácula por lo menos en un ojo. 


\section{Recomendaciones}

- No hay contraindicación de laserterapia durante el embarazo si la paciente lo requiere. Sin embargo, lo ideal es que se realice la evaluación oftalmológica previa a la concepción, lo cual reduce el riesgo de empeoramiento durante el embarazo.

- Tener presente que en pacientes con retinopatía proliferativa severa no tratada debe evitarse el parto vaginal con maniobra de Valsalva porque puede conducir a hemorragia retinal. En ese caso debe considerarse una cesárea programada.

Fuerza de la recomendación: alta. Calidad de la evidencia: fuerte a favor.

\section{Neuropatía diabética}

Considerar que no existen guías acerca del manejo de la neuropatía en el embarazo. Se aconseja descartar presencia de neuropatía en la consulta preconcepcional.

\section{Neuropatía autonómica}

Recomendaciones:

- Si la paciente presenta segmento QT prolongado en el ECG se sugiere realizar parto por cesárea.

- Si presenta labilidad glucémica por gastroenteropatía diabética se aconseja instruir a la paciente para que realice un plan alimentario fraccionado y utilice el esquema de insulinoterapia apropiado para esta complicación.

\section{C) CUIDADOS DURANTE EL PARTO}

\section{Manejo metabólico del trabajo del parto y la cesárea}

El manejo metabólico de las gestantes con diabetes pregestacional tiene como objetivo evitar las complicaciones maternas (cetosis de ayuno, hipoglucemia, hiperglucemia o cetoacidosis) y neonatales (hipoglucemia y distrés respiratorio).

\section{Recomendaciones}

Durante el trabajo de parto y la cesárea mantener la glucemia entre 80 y $120 \mathrm{mg} / \mathrm{dl}$.

Fuerza de la recomendación: alta. Calidad de la evidencia: fuerte a favor.

\section{Recomendaciones para el trabajo de parto o cesárea no programada}

- Realizar un aporte intravenoso de solución glucosada al $5 \%$ a 35 gotas por minuto ( $5 \mathrm{~g} /$ hora) según los niveles glucémicos y si se aplicó o no la dosis habitual de insulina basal. Regular el goteo según requerimiento.

- Controlar la glucemia cada 2 hs hasta que comience el trabajo de parto activo, y luego en forma horaria hasta el parto o cesárea.

- Aplicar insulina regular o análogo rápido subcutáneo cada 3 hs si lo requiere.

- Considerar según el caso individual si se requiere insulina basal (administrar un tercio o la mitad de dosis habitual).

- Si es posible utilizar infusión de insulina intravenosa continua de insulina; se sugiere la siguiente metodología:

- Iniciar infusión de insulina si la glucemia es mayor de $120 \mathrm{mg} / \mathrm{dl}$. Infusión endovenosa de insulina inicial: 0,5 a $1 \mathrm{UI} /$ hora.

- Si la glucemia es menor de $70 \mathrm{mg} / \mathrm{dl}$ reducir (o cerrar) el goteo de insulina o aumentar el aporte de glucosa intravenosa utilizando dextrosa al $10 \%$.

- Si la glucemia se mantiene entre 80 y $120 \mathrm{mg} / \mathrm{dl}$, continuar con el mismo aporte de glucosa e insulina.

- En caso de utilizar infusión continua de insulina, suspender luego del alumbramiento.

Fuerza de la recomendación: moderada. Calidad de la evidencia: débil a favor. 


\section{Recomendaciones para la cesárea programada}

- Convenientemente realizar la cesárea a primera hora de la mañana.

- Suspender la dosis matinal de insulina.

- Con más de 6 hs de ayuno, colocar infusión de dextrosa al $5 \%$.

- Realizar control glucémico cada 2 hs.

- Si la glucemia es mayor a 120 mg/dl, iniciar insulinoterapia como se describió en el apartado anterior.

- En caso de utilizar infusión continua de insulina, suspender luego del alumbramiento.

- Continuar con aporte de dextrosa al 5\% hasta el inicio de la alimentación.

- Controlar la glucemia cada 4 hs hasta iniciar la ingesta, y luego pre y postprandiales.

- Aplicar insulina regular o análogo rápido subcutáneo si la glucemia es mayor a 120 $\mathrm{mg} / \mathrm{dl}$ durante el ayuno y preprandial al iniciar la ingesta.

- Recordar que la mayoría de las pacientes con DM1 no tiene requerimiento de insulina durante las primeras 24 hs posteriores al parto, por lo cual se recomienda sólo correcciones con insulinas prandiales.

- Comenzar con insulina basal cuando el requerimiento diario de análogo o insulina regular sea superior a 10-15 unidades.

Fuerza de la recomendación: moderado. Calidad de la evidencia: fuerte a favor.

\section{Manejo obstétrico}

\section{Uso de corticoides en la maduración pulmonar}

Los corticoides se emplean en el embarazo para acelerar la maduración pulmonar fetal, disminuir la mortalidad perinatal y la hemorragia intraventricular cuando existe riesgo de nacimiento antes de la semana 34.

Considerar que:

- En mujeres con diabetes el riesgo de parto prematuro puede estar aumentado y que la maduración pulmonar fetal está retardada si no se logra la euglucemia.

- La hiperglucemia materna favorece la hiperglucemia e hiperinsulinemia fetal. Ésta inhibe el cortisol y disminuye la producción de surfactante pulmonar, cuya función es madurar el pulmón fetal.

- El surfactante pulmonar comienza a producir- se entre la semana 22 y 24, sin embargo su función activa es adecuada a partir de las 35 a 36 semanas.

- Uno de los efectos colaterales de los corticoides es la hiperglucemia. En mujeres con diabetes no está contraindicado su uso como tratamiento antenatal para madurar el pulmón fetal, excepto cuando la paciente está descompensada o inmunocomprometida.

- Para garantizar el buen control y evitar las hiperglucemias transitorias es necesario incrementar la frecuencia del automonitoreo glucémico, aumentar el aporte de insulina y contar con un grupo experimentado de profesionales

- Para prescribir maduración pulmonar con corticoides en embarazadas con diabetes se requiere un abordaje individualizado donde se evalúan los riesgos y beneficios de cada situación clínica particular, la frecuencia de los controles de glucemia (7 ó más/día) y el aumento del requerimiento de insulina. Cualquier protocolo que prevenga la hiperglucemia pre y post prandial es válido.

Se recomienda:

- Utilizar glucocorticoides que no sean de depósito, $12 \mathrm{mg}$ intramuscular de betametasona cada 24 hs en dos dosis o 6 mg intramuscular de dexametasona cada 12 hs durante dos días (cuatro dosis).

- Realizar la maduración con las embarazadas internadas.

- Mantener la glucemia entre 80 y 120 mg/dl.

\section{Criterios de internación}

Considerar internación en los siguientes casos:

- Asociación con estados hipertensivos del embarazo.

- Deterioro de la función renal.

- Deterioro del control metabólico.

- Sospecha de alteración de la salud fetal.

- En caso que la paciente lo requiera por situación social de riesgo.

- Si las normas de atención de pacientes diabéticas de la institución lo indican.

- Causa obstétrica que indique riesgo de nacimiento prematuro (amenaza de parto prematuro, restricción de crecimiento, metrorragia de la segunda mitad, etc).

\section{D) CUIDADOS POSTNATALES}

Las mujeres diabéticas en tratamiento con insulina tienen riesgo incrementado de desarrollar hipoglucemia luego del parto. El recién nacido presenta elevado riesgo de requerir cuidados intensivos. 
Se recomienda:

- Sugerir a todas las pacientes con diabetes pregestacional tener el parto en un centro de atención donde exista una unidad de cuidados intensivos neonatales.

- Luego del parto en las mujeres con diabetes pregestacional reducir inmediatamente la dosis de insulina y monitorear de cerca los niveles de glucosa en sangre y adecuar la dosis de insulina.

- Realizar el control de la glucemia en todos los recién nacidos de mujeres con diabetes debido al mayor riesgo de hipoglucemia neonatal.

- Referir a las pacientes con diabetes pregestacional a los cuidados previos al embarazo respecto de la diabetes.

- Dado que la actividad física en el puerperio reduce la aparición de la DM2 se recomienda aconsejar la continuidad o el inicio de un programa de ejercicio en esta etapa.

\section{Vacunación}

Durante el puerperio es importante tener el esquema de vacunación completo porque además de cuidar a la madre también se protege al bebé.

Se recomienda:

- Inmunizar contra la influenza si la paciente no recibió esta vacuna durante el embarazo.

- Administrar vacuna DPT si la paciente no la recibió durante el embarazo

- Administrar la primera dosis de la vacuna de la varicela y, siempre que sea posible, antes de ser dada de alta del hospital en las mujeres en las que no existe evidencia de inmunización contra el virus. La segunda dosis de la vacuna se administra entre las cuatro y ocho semanas después de la primera dosis.

\section{Lactancia}

La lactancia facilita en la madre la reducción del peso postparto, también disminuye el riesgo materno y del recién nacido de desarrollar en el futuro DM2, intolerancia a la glucosa y obesidad.

Se recomienda:

- Considerando que existe mayor riesgo de hipoglucemia en el período postparto inmediato, se aconseja que las mujeres que están en tratamiento con insulina tengan una colación disponible antes o durante el amamantamiento.

- No usar hipoglucemiantes orales durante la lactancia considerando la falta de evidencia disponible al respecto, si bien la concentración de met- formina en leche materna es baja $(0,28-1,08 \%)$ y la concentración de sulfonilurea es mínima.

\section{Recomendaciones para la cesárea programada}

- La lactancia materna confiere beneficios metabólicos a largo plazo para la madre y el recién nacido.

- Evitar durante la lactancia las medicaciones para el tratamiento de las complicaciones de la diabetes que fueron discontinuadas en el período preconcepcional.

- Considerando los beneficios nutricionales e inmunológicos, se recomienda promover la lactancia y que toda mujer con o sin diabetes intente amamantar.

Fuerza de la recomendación: alta. Calidad de la evidencia: fuerte a favor.

\section{Anticoncepción}

Se recomienda:

- La planificación familiar y el uso de métodos anticonceptivos cumplen un rol fundamental en la consulta de todas las mujeres diabéticas en edad fértil (desde la menarca hasta el climaterio).

- La principal herramienta para evitar el embarazo no planificado es una adecuada consejería en salud sexual y reproductiva, dispositivo de atención que implica el asesoramiento personalizado realizado por personal capacitado, con el objetivo de acompañar a las personas en la toma de decisiones autónomas sobre su salud sexual y reproductiva.

- Es relevante considerar la situación singular de cada persona y buscar junto con ella el método más adecuado según sus preferencias, costumbres y estado de salud.

- El diabetólogo, médico de cabecera de estas mujeres, debe incluir la consejería en anticoncepción en la consulta habitual, aunque sea el ginecólogo quien finalmente provea el método. El empoderamiento de estas mujeres por su médico de cabecera redunda en beneficio de la salud reproductiva y disminuye las complicaciones asociadas al embarazo no planificado o sin optimización metabólica.

- En la elección del método anticonceptivo deben considerarse los Criterios Médicos de Elegibilidad para el Uso de Anticonceptivos de la OMS indicados en el Anexo II.

- Considerar además los siguientes métodos: 
- Barrera: siempre recordar a las usuarias que los preservativos son los únicos métodos anticonceptivos que previenen las enfermedades de transmisión sexual, incluidas el VIH/Sida.

- Anticoncepción hormonal de emergencia (AHE): es un método hormonal de progestágeno (levonorgestrel) para utilizar en forma posterior a una relación sexual sin protección o cuando falló el método que estaba usándose. El principal mecanismo anticonceptivo de la AHE de levonorgestrel es la anovulación. Asimismo provoca un espesamiento del moco del cuello del útero, lo que dificulta el ascenso de los espermatozoides a las trompas de Falopio, sitio donde se produce la fecundación. No tiene contraindicaciones. En caso que el embarazo ya se haya producido, la AHE no lo interrumpe, es decir, no provoca un aborto y tampoco produce defectos congénitos.

- Procedimientos de esterilización quirúrgica: dada la irreversibilidad o permanencia de los procedimientos de esterilización debe tenerse especial cuidado en asegurar una elección voluntaria informada del método por parte del usuario o de la usuaria. Tener en cuenta las comorbilidades determinantes del riesgo prequirúrgico.

Toda paciente con diagnóstico de diabetes debe recibir adecuada consejería acerca de un método anticonceptivo y utilizar aquel más seguro hasta lograr los objetivos metabólicos.

\section{CONCLUSIONES}

Las presentes recomendaciones fueron elaboradas en el marco del Comité de Diabetes y Embarazo de la Sociedad Argentina de Diabetes con el fin de cuidar la salud metabólica y reproductiva de la mujer con diabetes pregestacional, programar sus embarazos y prevenir complicaciones.

En la elaboración de estas recomendaciones se utilizó el sistema GRADE¹. Para ello, se evaluó la literatura existente, incluyendo guías previas de la Sociedad Argentina de Diabetes ${ }^{2}$ y del Ministerio de Salud de la Nación ${ }^{3-5}$, guías elaboradas y recomendaciones de diversas Sociedades Internacionales vinculadas al cuidado de la salud de gestantes ${ }^{6-22}$, como así también estudios realizados por grupos expertos en áreas específicas que incluyen el control preconcepcional ${ }^{23}$, el control metabólico ${ }^{24-26}$, la insulinoterapia ${ }^{27-33}$, los hipoglu- cemiantes orales ${ }^{34-37}$, las inmunizaciones ${ }^{38-40}$, el control nutricional41-44, cambios de estilo de vida ${ }^{45-46}$, bases y complicaciones ${ }^{47-50}$, la hipertensión arterial ${ }^{51-52}$, los cuidados obstétricos ${ }^{53-54}$ y postnatales ${ }^{55-56}$, entre otros.

A partir de la revisión y discusión de estos estudios y observaciones se consensuaron las recomendaciones presentadas. Esperamos que las mismas resulten de ayuda al momento de tomar decisiones clínicas, sin sustituir en ningún caso el juicio clínico, y contribuyan a mejorar la salud de las mujeres con diabetes pregestacional.

\section{REFERENCIAS}

1. Neuman I, PantojaT, Peñaloza B, Cifuentes L, Rada G. El sistema GRADE: un cambio en la forma de evaluar la calidad de la evidencia y la fuerza de las recomendaciones. Revista Med Chile 2014; 142:630-635

2. Faingold MC, Lamela C, Gheggi M, Lapertosa S, Di Marco I, Basualdo MN, Rovira G, et al. Recomendaciones para gestantes con diabetes pregestacional. Rev Soc Arg Diab 2010; 44(2):93-107.

3. Ministerio de Salud. Presidencia de la Nación Argentina. Guía para el diagnóstico y tratamiento de la hipertensión en el embarazo; 2010.

4. Ministerio de Salud. Presidencia de la Nación Argentina. Métodos anticonceptivos. Guía práctica para profesionales de la salud; 2012.

5. Ministerio de Salud. Presidencia de la Nación Argentina. Recomendaciones para la Práctica del Control preconcepcional, prenatal y puerperal. Dirección Nacional de Maternidad e Infancia; 2013.

6. ACOG. Commitee Opinion 560. Medically indicated late-preterm and early-term deliveries. Obstet Gynecol 2013; 121:908.

7. American Diabetes Association. Management of diabetes in pregnancy. Diabetes Care 2016; 39(1):94-98.

8. American Diabetes Association. Management of diabetes in pregnancy. Diabetes Care 2015; 38(1):77-79.

9. American Diabetes Association Guide 2012. Nutrition Therapy for Diabetes. Second Edition.

10. Bailey TS, Grunberger G, Bode BW, Handelsman Y, Hirsch IB, Jovanovič L, Lawrence Roberts V, Rodbard V, Tamborlane WV, Walsh J. AACE and ACE 2016 outpatient CGM Consensus Statement. Endocr Pract 2016; 22:231-261.

11. Blumer I, Hadar E, Hadden DR, Jovanovič L, Mestman JH, Murad $\mathrm{MH}$, Yogev $\mathrm{Y}$. Diabetes and pregnancy: an endocrine society clinical practice guideline. J Clin Endocrinol Metab, 2013; 98(11):4227-4249.

12. Canadian Agency for Drug and Technologies in Health. Intravenous Insulin Infusions in Labouring Women: Guidelines. 2011. Disponible en: https://www.cadth.ca/media/pdf/DM/Rapid_Response_Insulin_Infusions_During_Labour_e.pdf.

13. Canadian Diabetes Association Clinical Practice Guidelines Expert Committee. Clinical Practice Guidelines for the prevention and management of Diabetes in Canada. Canadian Journal of Diabetes 2013; 37(Suppl1):1-212.

14. Guideline Development Group. Management of diabetes from preconception to the postnatal period: summary of NICE guidance. BMJ 2008; 336(7646):714-717. 
15. National Collaborating Centre for Women's and Children's Health (UK). Diabetes in pregnancy: management of diabetes and its complications from preconception to the postnatal period. Intrapartum care. National Institute for Health and Care Excellence (UK) 2015; NICE Guideline, N³. Disponible en: http://nice.org.uk/ guidance/ng3.

16. Organización Mundial de la Salud. Criterios médicos de elegibilidad para el uso de anticonceptivos. Cuarta Edición. 2009. Disponible en: http://apps.who.int/iris/bitstre am/10665/44710/1/9789243563886_spa.pdf.

17. Organización Mundial de la Salud. Criterios médicos de elegibilidad para el uso de anticonceptivos. Quinta Edición. 2015. Disponible en: http://apps.who.int/iris/bitstream/10665/205016/1/ WHO_RHR_15.07_spa.pdf?ua=1.

18. Organización Mundial de la Salud. Recomendaciones OMS para la prevención y el tratamiento de la preeclampsia y eclampsia. 2013. Disponible en: http://apps.who.int/iris/bitstream/10665/119742/1/WHO_RHR_14.17_spa.pdf.

19. Ottawa $(\mathrm{ON})$ : Canadian Agency for Drugs and Technologies in Health. Insulin pumps for adults with type 1 diabetes: a review of clinical effectiveness, cost-effectiveness and guidelines 2015. Disponible en: https://www.cadth.ca/insulin-pumps-adults-type1-diabetes-review-clinical-effectiveness-cost-effectiveness-andguidelines.

20. Shields L, Tsay GS. California diabetes and pregnancy program sweet success guidelines for care. California Department of Public Health website. Revised July 2012. Disponible en: http:// www.cdappsweetsuccess.org/Portals/0/Guidelines/Guidelines for Care_All 1.

21. Thompson D, Berger H, Feig D, Gagnon R, KaderT, Keely E, Kozak $S$, et al. Clinical practice guidelines diabetes and pregnancy. Can J Diabetes 2013; 37 (Suppl 1):168-183

22. Rasmussen $\mathrm{KL}$, Yaktine A. Weight gain during pregnancy. Reexamining the Guidelines. Institute of Medicine (US) and National Research Council (US) Committee to Reexamine IOM Pregnancy Weight Guidelines.Washington (DC): National Academies Press (US); 2009.ISBN-13: 978-0-309-13113.

23. Jensen DM, Korsholm L, Ovesen P, Beck-Nielsen H, Moelsted-Pedersen L, Westergaard JG, Moeller M, Damm P. Peri-conceptional A1C and risk of serious adverse pregnancy outcome in 933 women with type 1 diabetes. Diabetes Care 2009; 32:1046-1048.

24. Feig DS, Donovan LE, Corcoy R, Murphy KE, Amiel SA, Hunt KF, Asztalos E, Barrett JFR, Sanchez JJ, de Leiva A, Hod M, Jovanovic L, Keely E, McManus R, Hutton EK, Meek CL, Stewart ZA, Wysocki T, O'Brien R, Ruedy K, Kollman C,Tomlinson G, Murphy HR; CONCEPTT Collaborative Group. Continuous glucose monitoring in pregnant women with type 1 diabetes (CONCEPTT): a multicentre international randomised controlled trial. Lancet. 2017 Sep 14. pii: S0140-6736(17)32400-5. doi: 10.1016/S0140-6736(17)32400-5.

25. Hernandez TL, Friedman JE, Van Pelt RE, Barbour LA. Patterns of glycemia in normal pregnancy should the current therapeutic targets be challenged? Diabetes Care 2011; 34(7): 1660-1668.

26. Rebolledo OR, Actis Dato SM, Gagliardino JJ. Niveles de fructosamina en embarazadas con distinta edad de gestación y niños de primera y segunda infancia. Rev Soc Argent Diab 1997; 31(4):131-9.

27. Drever E, Tomlinson G, Bai AD, Feig DS. Insulin pump use compared with intravenous insulin during labour and delivery: the INSPIRED observational cohort study. Diabet Med 2016; 33(9):1253-1259.

28. Farrar D, Tuffnell DJ, West J, West HM. Continuous subcutaneous insulin infusion versus multiple daily injections of insulin for pregnant women with diabetes. Cochrane Database of Systematic Reviews 2016; (6):CD005542.
29. Heller S, Damm P, Mersebach H, Skjøth TV, Kaaja i, Hod M, Durán-García $\mathrm{S}$, et al. Hypoglycemia in type 1 diabetic pregnancy: role of preconception insulin aspart treatment in a randomized study. Diabetes Care 2010; 33(3):473-477.

30. Hod M, Mathiesen ER, Jovanovič L, McCance DR, Ivanisevic M, Durán-García S, Brøndsted L, et al. A randomized trial comparing perinatal outcomes using insulin detemir or neutral protamine Hagedorn in type 1 diabetes. J Matern Fetal Neonatal Med 2014; 27(1):7-13.

31. Mathiesen ER, Kinsley B, Amiel SA, Heller S, McCance D, Duran S, Bellaire S, Raben A; Insulin Aspart Pregnancy Study Group. Maternal glycemic control and hypoglycemia in type 1 diabetic pregnancy: a randomized trial of insulin aspart versus human insulin in 322 pregnant women. Diabetes Care 2007; 30(4):771-6.

32. McElduff A, Moses RG. Insulin therapy in pregnancy. Endocrinol Metab Clin N Am 2012; 41(1):161-173.

33. Pollex E, Moretti ME, Koren G, Feig DS. Safety of insulin glargine use in pregnancy: a systematic review and meta-analysis. Ann Pharmacother 2011; 45(1):9-16.

34. Ibrahim MI, Hamdy A, Shafik A, Taha S, Anwar M, Faris M. The role of adding metformin in insulin-resistant diabetic pregnant women: a randomized controlled trial. Arch Gynecol Obstet 2014; 289(5):959-965.

35. Jiang YF, Chen XY, Ding T, Wang XF, Zhu ZN, Su SW. Comparative efficacy and safety of OADs in management of GDM: network meta-analysis of randomized controlled trials. J Clin Endocrinol Metab 2015; 100(5):2071-2080.

36. Rowan JA, Rush EC, Obolonkin V, Battin M, Wouldes T, Hague WM. Metformin in gestational diabetes: the offspring follow-up (MiG TOFU): body composition at 2 years of age. Diabetes Care 2011; 34(10):2279-84.

37. Rowan JA, Hague WM. Metformin versus insulin for the treatment of gestational diabetes. N Engl J Med 2008; 8 358(19):2003-15.

38. Regan AK. The safety of maternal immunization. Hum Vaccin Innmunother 2016; 19:1-5.

39. Swamy GK, Heine P. Vaccinations for pregnant women. Obstet Gynecol 2015; 125(1): 212-226.

40. Vizzotti C, Neyro S, Katz N, Juárez MV, Pérez Carrega ME, Aquino A, Kaski Fullone F. Maternal immunization in Argentina: a storyline from the prospective of a middle income country. Vaccine 2015; 33: 6413-6419.

41. Gunderson EP. Intensive nutrition therapy for gestational diabetes. Rationale and current issues. Diabetes Care 1997; 20(2):221-226.

42. Moreno-Castilla $C$, Hernández M, Bergua M, et al. Low-carbohydrate diet for the treatment of gestational diabetes: a randomized controlled trial. Diabetes Care 2013; 36:2233-8.

43. Calvo EB, López LB, Balmaceda $Y$, et al. Referente charts for weight gain and body mass index during pregnancy obtained from a healthy cohort. J Matern and Fetal \& Neonatal Medicine. 2009; 22 (1):36-42.

44. Tremblay A, Gilbert J. Milk products, insulin resistance syndrome and type 2 diabetes. J Am Coll Nutr 2009; 28 Suppl 1:S91-S102.

45. Anjana RM, Sudha V, Lakshmipriya N, Anitha C, Unnikrishnan R, Bhavadharini B, Mahalakshimi MM, et al. Physical activity patterns and gestational diabetes outcomes. The wings Project. Diabetes Res Clin Pract 2016; 116:253-262.

46. Koivusalo SB, Rönö K, Klemetti MM, Roine RP, Lindström J, Erkkola M, Kaaja RJ, et al. Gestational diabetes mellitus can be prevented by lifestyle intervention. The Finnish Gestational Diabetes Prevention Study (RADIEL): a randomized controlled trial. Diabetes Care 2016; 39(1):24-30. 
47. Rasmussen KL, Laugesen CS, Ringholm L, Vestgaard M, Damm $P$, Mathiesen ER. Progression of diabetic retinopathy during pregnancy in women with type 2 diabetes. Diabetologia 2010; 53(6):1076-1083.

48. Pallardo-Sánchez LF. Diabetes y embarazo. Editorial EDIKAMED. Edición 2008.

49. Sibai BM, Viteri OA. Diabetic ketoacidosis in pregnancy. Obstet Gynecol 2014; 123(1):167-178.

50. Simmons D. Diabetes and obesity in pregnancy. Best Pract Res Clin Obstet Gynaecol 2011; 25: 25-36.

51. Jensen DM, Damm P, Ovesen P, Mølsted-Pedersen L, BeckNielsen H, Westergaard JG, Moeller M, et al. Microalbuminuria, preeclampsia and preterm delivery in pregnancy with type 1 diabetes: results from a nationwide Danish study. Diabetes Care 2010; 33:90-94.

52. Mathiesen ER, Ringholm L, Feldt-Rasmussen B, Clausen $P$, Damm P. Obstetric nephrology: pregnancy in women with diabetic nephropathy. The role of antihypertensive treatment. Clin J Am Soc Nephrol 2012; 7(12): 2081-2088.
53. Cantwell R, Clutton-Brock T, Cooper G, Dawson A, Drife J, Ga$\operatorname{rrod} D$, Harper $A$, et al. Saving mothers' lives: reviewing maternal deaths to make motherhood safer: 2006-2008. The eighth report on confidential enquiries into maternal deaths in the United Kingdom. BJOG 2011; 118(1):1-203.

54. Roberts D, Brown J, Mendley N, Dalziel S. Antenatal corticosteroids for accelerating fetal lung maturation for women at risk of preterm birth. Cochrane Database Syst Rev 2017; 3: CD004454.

55. O'Reilly M, Avalos G, Dennedy MC, O'Sullivan EP, Dunne FP. Breast feeding is asssociated with reduced post partum maternal glucose intolerance after gestational diabetes. Ir Med J. 2012; 105(5):31-36.

56. Pereira PF, Alfenas R de C, Araujo RM. Does breathfeeding influence the risk of developing diabetes mellitus in children? A review of current evidence. J Pediatr (Rio J) 2014; 90(1):7-15.

\section{ANEXOS}

Anexo I

\section{Breve guía resumida. Consulta preconcepcional}

1. Informar a la paciente que planifica el embarazo sobre la repercusión del mismo en la evolución de su enfermedad y en los resultados perinatales.

2. Realizar anamnesis detallada para identificar riesgos y patologías asociadas.

3. Aconsejar sobre la importancia del control glucémico antes de la concepción.

4. El objetivo de control metabólico en el embarazo es lograr una Hb glicosilada menor a 7\%.

5. Realizar laboratorio preconcepcional según corresponda (serologías, tipificación sanguínea, ferremia, dosaje de vitamina B12), rastreo de enfermedad celíaca y tiroidea.

6. Efectuar pruebas de función renal y control oftalmológico.

7. Evaluar la función cardiovascular.

8. Realizar evaluación nutricional con el objetivo de lograr un peso saludable y/o descenso de peso.

9. Recomendar un plan de actividad física.

10. Suspender drogas desaconsejadas durante el embarazo: hipoglucemiantes orales, IECA, ARAll, estatinas, fibratos.

11. Suplementar con ácido fólico $5 \mathrm{mg}$.

12. Recomendar el uso de insulinas aprobadas en el embarazo.

13. Realizar evaluación ginecológica completa.

14. Efectuar evaluación odontológica.

\section{Anexo II}

Recomendaciones basadas en los Criterios Médicos de Elegibilidad para el Uso de Anticonceptivos de la Organización Mundial de la Salud, Quinta Edición, 2015

\begin{tabular}{|c|c|c|}
\hline Categoría & Situaciones clínicas de las pacientes & Criterio clínico para la práctica cotidiana \\
\hline 1 & $\begin{array}{l}\text { Una condición para la que no hay restricción en cuanto al } \\
\text { uso del método anticonceptivo }\end{array}$ & Use el método en cualquier circunstancia \\
\hline 2 & $\begin{array}{l}\text { Una condición donde las ventajas del uso del método gene- } \\
\text { ralmente superan los riesgos teóricos o probados }\end{array}$ & En general, use el método \\
\hline 3 & $\begin{array}{l}\text { Una condición donde los riesgos teóricos o probados gene- } \\
\text { ralmente superan las ventajas del uso del método }\end{array}$ & $\begin{array}{l}\text { El uso del método generalmente no se recomienda, a menos que otros } \\
\text { métodos más adecuados no estén disponibles o no sean } \\
\text { aceptados o haya serias dificultades para la adherencia }\end{array}$ \\
\hline 4 & $\begin{array}{l}\text { Una condición que representa un riesgo de salud } \\
\text { inadmisible si se utiliza el método anticonceptivo }\end{array}$ & Se desaconseja el uso del método \\
\hline
\end{tabular}

Tabla A: Categorías según situación clínica de la paciente. 


\begin{tabular}{|c|c|c|c|}
\hline $\begin{array}{l}\text { Criterios de Elegibilidad } \\
\text { de la OMS } 2015\end{array}$ & $\begin{array}{c}\text { Anticonceptivos } \\
\text { hormonales combinados } \\
\text { (AOC, PAC, AVC, AIC) }\end{array}$ & $\begin{array}{c}\text { Anticonceptivos de } \\
\text { progestina sola (AOPS, } \\
\text { AMPD Y EN-NET, LNG } \\
\text { Y ETG) }\end{array}$ & $\begin{array}{l}\text { Dispositivos intrauterinos } \\
\text { (DIU-CU y DIU-LNG) }\end{array}$ \\
\hline \multicolumn{4}{|l|}{ Diabetes } \\
\hline $\begin{array}{l}\text { Sin enfermedad vascular (independientemente } \\
\text { del uso de insulina) }\end{array}$ & 2 & 2 & $\begin{array}{l}\text { Cu: } 1 \\
\text { LNG: } 2\end{array}$ \\
\hline Neuropatía/ retinopatía/ nefropatía & $3 / 4$ & $\begin{array}{l}2 \\
\text { Excepto AMPD y } \\
\text { EN-ET: } 3\end{array}$ & $\begin{array}{l}\text { Cu: } 1 \\
\text { LNG: } 2\end{array}$ \\
\hline $\begin{array}{l}\text { Otra enfermedad vascular o diabetes de más de } \\
20 \text { años de evolución }\end{array}$ & $3 / 4$ & $\begin{array}{l}2 \\
\text { Excepto AMPD y } \\
\text { EN-ET: } 3\end{array}$ & $\begin{array}{l}\text { Cu: } 1 \\
\text { LNG: } 2\end{array}$ \\
\hline
\end{tabular}

Referencias: anticonceptivos orales combinados de dosis bajas (AOC); parche combinado (PAC); anillo vaginal combinado (AVC); anticonceptivos inyectables combinados (AIC); anticonceptivos orales de progestina sola (AOPS); acetato de medroxiprogesterona de depósito (AMPD); enantato de noretisterona (EN-NET); implantes de levonorgestrel (LNG) y etonogestrel (ETG); píldoras anticonceptivas de emergencia (PAE); dispositivos intrauterinos con cobre (DIU-Cu); DIU liberadores de levonorgestrel (DIU-LNG).

Tabla B: Criterios de eligibilidad de anticonceptivos en pacientes con diabetes.

\begin{tabular}{|c|c|c|c|}
\hline $\begin{array}{l}\text { Criterios de Elegibilidad } \\
\text { de la OMS } 2015\end{array}$ & $\begin{array}{l}\text { Anticonceptivos } \\
\text { hormonales combinados } \\
\text { (AOC, PAC, AVC, AIC) }\end{array}$ & $\begin{array}{c}\text { Anticonceptivos de } \\
\text { progestina sola (AOPS, } \\
\text { AMPD Y EN-NET, LNG } \\
\text { Y ETG) }\end{array}$ & $\begin{array}{l}\text { Dispositivos intrauterinos } \\
\text { (DIU-CU y DIU-LNG) }\end{array}$ \\
\hline Obesidad (IMC>=30) & 1 & 1 & 1 \\
\hline \multicolumn{4}{|l|}{ Hipertensión } \\
\hline $\begin{array}{l}\text { Historia de presión alta, sin posibilidad de } \\
\text { evaluar la tensión arterial }\end{array}$ & 3 & 2 & $\begin{array}{l}\text { Cu: } 1 \\
\text { LNG: } 2\end{array}$ \\
\hline $\begin{array}{l}\text { Antecedentes de hipertensión, con presión } \\
\text { controlada y posibilidad de evaluarla }\end{array}$ & 3 & $\begin{array}{l}1 \text { Excepto AMPD y } \\
\text { EN-NET: } 2\end{array}$ & 1 \\
\hline Máxima entre 140 y 159, mínima entre 90 y 99 & 3 & $\begin{array}{l}1 \text { Excepto AMPD y } \\
\text { EN-NET: } 2\end{array}$ & 1 \\
\hline $\begin{array}{l}\text { Máxima mayor de } 160 \text { y/o mínima mayor } \\
\text { de } 100\end{array}$ & 4 & $\begin{array}{l}2 \text { Excepto AMPD y } \\
\text { EN-NET: } 3\end{array}$ & $\begin{array}{l}\text { Cu: } 1 \\
\text { LNG: } 2\end{array}$ \\
\hline Enfermedad vascular & 4 & $\begin{array}{l}2 \text { Excepto AMPD y } \\
\text { EN-NET: } 3\end{array}$ & $\begin{array}{l}\text { Cu: } 1 \\
\text { LNG: } 2\end{array}$ \\
\hline $\begin{array}{l}\text { Múltiples factores de riesgo de enfermedad } \\
\text { cardiovascular (edad mayor, fumar, diabetes, } \\
\text { hipertensión, dislipemias) }\end{array}$ & $3 / 4$ & $\begin{array}{l}2 \text { Excepto AMPD y } \\
\text { EN-NET: } 3\end{array}$ & $\begin{array}{l}\text { Cu: } 1 \\
\text { LNG: } 2\end{array}$ \\
\hline $\begin{array}{l}\text { Cardiopatía isquémica (enfermedad actual } \\
\text { o historia) }\end{array}$ & 4 & $\begin{array}{l}\text { Iniciación: } 2 \text { excepto AMPD } \\
\text { y EN-NET: } 3 \\
\text { Continuación: } 3\end{array}$ & $\begin{array}{l}\text { Cu: } 1 \\
\text { LNG: } 2 \text { inicio y } \\
3 \text { continuación }\end{array}$ \\
\hline $\begin{array}{l}\text { Accidente cerebrovascular } \\
\text { (historia de accidente cerebrovascular) }\end{array}$ & 4 & $\begin{array}{l}\text { Iniciación: } 2 \text { excepto AMPD } \\
\text { y EN-NET:3 } \\
\text { Continuación: } 3\end{array}$ & $\begin{array}{l}\text { Cu: } 1 \\
\text { LNG: } 2\end{array}$ \\
\hline
\end{tabular}

Tabla C: Criterios de eligibilidad de anticonceptivos en pacientes con comorbilidades. 


\section{Anexo III \\ Breve guía resumida. Seguimiento durante el embarazo}

1. El seguimiento durante el embarazo debería ser semanal hasta lograr metas de control metabólico, luego cada 15 días hasta la semana 32 y semanal hasta la internación.

2. Evaluar el laboratorio completo de la primera consulta.

3. Evaluación clínica: anamnesis alimentaria y examen físico (peso, TA, evaluación de presencia de edemas, etc).

4. Control nutricional de la ganancia de peso según el IMC inicial y de la cetonuria en ayunas.

5. Evaluación del perfil metabólico según el automonitoreo glucémico capilar pre y postprandial, la fructosamina cada tres semanas y la HbA1c mensual.

6. Evaluar la función renal y urocultivo en cada trimestre.

7. Realizar control oftalmológico en cada trimestre.

8. Efecuar control odontológico.

9. Realizar control ginecológico.

10. Recomendar vacunas influenza y DTP (luego de la semana 20), evaluar requerimientos de otras vacunas según cada paciente. 